\title{
Design and Implement an Exoskeleton Arm for Reinforcement the Human Muscles after Stroke
}

\author{
Duha Qais AbdulAmir ${ }^{1}$, Auns Qusai Hashim², Abdulnasir Hussin Ameer ${ }^{3}$
}

\section{Authors affiliations: \\ 1) Biomedical Engineering Department, Al-Nahrain University, Baghdad-Iraq. duha.qais@yahoo.com}

2) Biomedical Engineering Department, Al-Nahrain University, Baghdad-Iraq. uns alneami@yahoo.com

3) College of Medicine, University of Baghdad, Baghdad-Iraq.

Nasiramer@gmail.com

\section{Paper History:}

Received: $8^{\text {th }}$ April 2019

Revised: 12 ${ }^{\text {th }}$ May 2019

Accepted: $18^{\text {th }}$ Nov. 2019

\begin{abstract}
Mobility limitations in stroke survivors yield negative impacts on the quality of life for such individuals. Rehabilitation is needed to help them recover and regain mobility. Accordingly, this study aims to design and validate a "Robotic Exoskeleton" intended for stroke rehabilitation. The basic principles of this robotic exoskeleton device are its dependence on electromyography signal and electronic microcontroller to provide an efficient physiotherapy exercises system.

The robotic exoskeleton is a one degree of freedom which performs the flexion and extension of the elbow joint. After the design was completed, 19 subjects participated in this study: 4 healthy subjects, and 15 post-stroke patients.

The results showed the benefit of robotic exoskeleton in increasing the elbow range of motion, where angle of elbow flexion was raised from the first physiotherapy session to maximum elbow flexion in the last session.
\end{abstract}

Keywords: Exoskeleton, Stroke, Physiotherapy, Electrode, MyoWare.

$$
\begin{aligned}
& \text { تصميم و تطبيق مسند خارجي للذراع لتعزيز العضلات البشرية بعد السكتة } \\
& \text { اللماغية } \\
& \text { ضحى قيس عبدالأمير ، أنس قصي هاشم ، عبدالناصر حسين أمير } \\
& \text { يؤدي تحدد الحركة في مرضى السكنة الدماغية إلى تأثيرات سلبية على نوعية الحياة لهؤلاء الأفراد. هناك حاجة }
\end{aligned}
$$

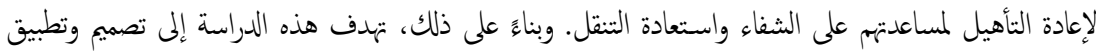

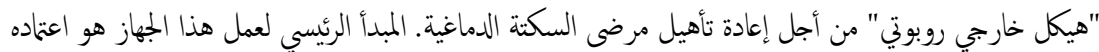

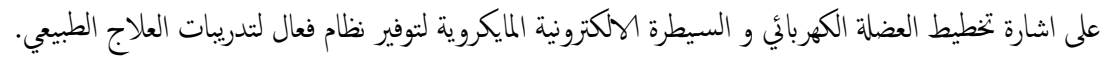

$$
\begin{aligned}
& \text { يؤدي جهاز المسند الخارجي حركة واحدة وهي حركة الثني والمد لمفصل المرفق. بعد ان اكتمل تصميم الجهاز ، } \\
& \text { شارك } 19 \text { شخصًا في هذه الدراسة: } 4 \text { أشخاص أصحاء و } 15 \text { مريضًا بعد السكتة المُ الدماغية. }
\end{aligned}
$$

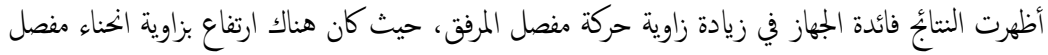

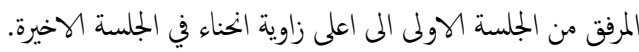

\section{Introduction}

There is a large number of people with movement disabilities who are unable to do their daily activities by themself. These weak movements are caused by several reasons such as injuries and surgeries, stroke, Traumatic Brain Injury (TBI), Spinal Cord Injury (SCI), and various neurological disorders. Many researchers are working on rehabilitation procedures for the therapy, to give the hope of independent life to these people.

Moreover, any injury that hinders a part of the body's movement leads to an unwanted reduction in life communication [2].
Alongside different statistical reports on brain injuries, there is a considerable encouragement to improve the rehabilitation of these patients (specifically stroke survivors). Through health care, it has been found that physiotherapy retained the best results in treating movement disorders, However, this treatment is associated with long working hours and impose a great burden on the therapist. Therefore, studies have sought to develop optimum care systems to reduce the costs and time associated with treatment. This optimum rehabilitation could be accomplished through the application of new technologies [1]. 
The avoidance of using a weak arm leads to learning not to neglect it, which complicates the process of rehabilitation of the weak limb and delay the treatment process, especially for patients with stroke. There is a significant development of some of the structural systems that support the weak limb and provide the necessary force and torque of the movement for individuals that would make them capable of working and assisting themselves. These systems are professionally designed with suitable measurements for human wear and are suitable for many medical, military and industrial applications. Because they are complex systems, they need skill in managing them, as well as being very expensive [3].

The cost is obviously the main factor in making advanced rehabilitation devices of limited use. As the science progresses, studies are being carried out to reduce the size of the medical rehabilitation equipment, thus reducing the cost and size, making it portable and easy to store, as well as being available to the patient to perform physical therapy at home [4].

Some topics include a survey of studying exoskeleton arm of the current state for upper limb disorders.

In 2013, Marco C. et al. [5], developed a modified portable robotic elbow exoskeleton (NEUROExos), designed for handling of stroke survivors in acute/sub-acute cases. Series Elastic Actuation (SEA) system was used in the design. Spring deformation was measured by two absolute encoders and the spring stiffness was recognized for the torque applied to the joint.

In 2014, Rodrigo C. et al. [6] suggested that the dynamic orthosis can have positive outcomes related to with the efficiency of functional tasks concerning the advancement of upper extremity functional capacity. It consisted of an exoskeleton, static orthosis, functional glove, control unit, and electromechanical actuators. The exoskeleton constituted of two nylon segments that is placed between the arm and forearm and linked by a pivot shaft. The results suggested that the system may support the functional rehabilitation of patients with hemiparesis.

In 2016, Christoffer B. et al. [7] constructed fullsize prototypes exoskeletons were in an iterative process to find the optimal structural solution. Lowcost materials were used. A servo motor was used to simulate human arm movement. The result was a construction controlled by evaluating the torques from the user's movement using of force sensors placed at the wrist.

In 2017, Borna Ghannadi [1] developed a highfidelity biomechatronic model of the human-robot interaction. The rehabilitation robot is a two degree of freedom (DOF) parallelogram linkage with joint friction and backlash, as well as nonlinear dynamics. Different musculoskeletal upper extremity, biomechanics, models are used to model human body motions while interacting with the rehabilitation robot model. Borna G. used the model-based control structures to provide biofeedback during a rehabilitation therapy. He focused on the modeling and control of an upper extremity rehabilitation robot interacting with musculoskeletal upper extremity models.

The Aims of the Present Study Include:

- Design the appropriate exoskeleton arm device based on the electrical muscle signal.

- Apply the system to the specific group of patients; stroke patients.

- Enable the patient to regularly receive treatment exercises without the continuous assistance of the therapist, as well as reduce the time and cost to enable the therapist to supervise several patients at the same time.

\section{Elbow Biomechanics}

The radius and the ulna in the forearm are linked with the humerus of the upper arm, as shown in (Figure 1). The humeroradial joint may be classified as a ball-and-socket joint with $3 \mathrm{DOF}$, whereas the humeroulnar joint is characterized as a hinge joint with $1 \mathrm{DOF}$. The connection of the bones forms a closed kinematic chain, limiting the elbow joint movement to $2 \mathrm{DOF}$ in general [8].

Flexion and extension occur in the sagittal plane. Flexion is movement in which the angle between the bones and body parts reduces. Whereas extension is the movement in which this angle increases [9].

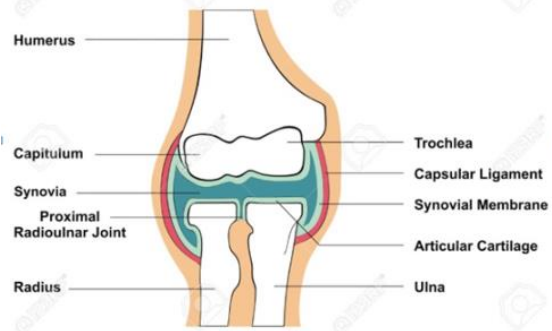

Figure (1): Elbow joint [8].

The exoskeleton, in this study, is 1 DOF supported the flexion-extension movement of the elbow joint.

\section{The Nature of Disability}

Stroke is the most common cause of disability. Although the mechanism of injury varies, stroke includes lesions in an individual's nervous system tissue. Strokes, also known as cerebrovascular accident (CVA), have become one of the most popular diseases which influence many patients rendering them unable to move or, in some cases, lead them to die in an increasingly aged community. WHO (World Health Organization) enumerates that annually 15 million people suffer a stroke around the world [10].

A motor dysfunction may occur depending on the position of the damaged tissue. There are two main types of strokes: hemorrhagic due to bleeding, and ischemic due to the lack of blood flow. Signs and symptoms of a stroke may involve inability to walk, move the arm or both limbs on one side of the body, feeling of imbalance, problems in speaking or understanding, or poor vision in one eye more than the other [11] 


\section{The Exoskeleton System and Rehabilitation}

An exoskeleton is an external structural mechanism whose joints correspond to those of the human body. The name stems from the words exterior and skeleton [12].

It is a kind of robotic devices and a humanmachine system that is focused on the human user [13]. The exoskeleton has the potential to enhance the machine's intelligence and strength in the human user by incorporating the intelligence of the human user and the power of the machine.

The first time the exoskeleton was used it was to support an animal to perform movement, protection, sensation and excretion functions [14].

The exoskeleton must be designed for comfortable and easy wearing so to remove intervention during upper-limb movement of the exoskeleton and the employer; cables, pulleys, links, motors, and other mechanical components of the exoskeleton must be positioned. For suitable control of the exoskeleton, mostly the number of degrees of freedom is similar to the number of mobilized joints [15].

If exoskeleton is applied in medical rehabilitation, it should be able to perform the movements repeatedly for the patient as the treatment received by the therapist [16].

\section{Materials and Methods}

The materials used in this study are:-

1) EMG Sensor, 2) Electrodes, 3) Gyroscope sensor, 4) Microcontroller, 5) Actuators, 6) Motor, 7) Motor drive, 8) Pulley, 9) Bowden cable, 10) Battery, 11) Charger, and 12) Switches.

- To pick out the surface electromyography EMG signal, MyoWare sensor was used to measure a muscle's activity as shown in (Figure 2), which was prepared to be suitable for wear and to have adaptable gain, the voltage supply is +5 Volts, current supply $9 \mathrm{~mA}$ with polarity of inversion preservation.

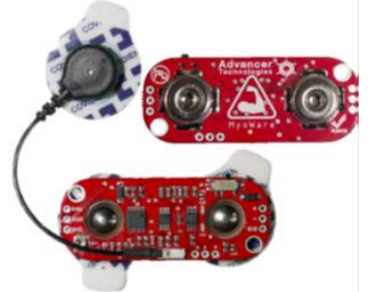

Figure (2): MyoWare sensor.

- The MyoWare sensor measures and amplifies the electrical activity from the muscle that is an analog signal that is easily converted to digital signal by the controller with an analog to digital converter.

- When the magnitude of the EMG signal is exactly denoted to the activity force from the muscle, it is utilized to determine the force signal sent to the exoskeleton.

- In this study integrated EMG signal shown in (Figure 3) was used for reading muscle activity and as an input signal to the microcontroller.

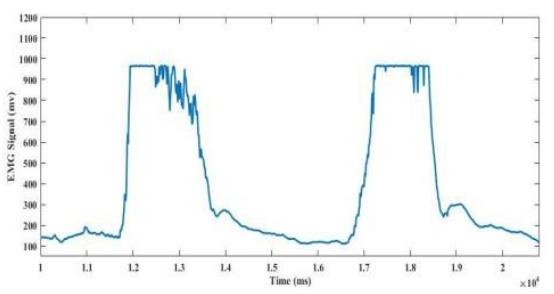

Figure (3): Integrated EMG signal.

- EMG signal act as calibration for each patient according to patients muscle activity.

- The microcontroller (Arduino Uno) reads this EMG signal through the analog input port (A0) and then it switch on the motor by sending on pulse through the digital output port ( 2 and 3 ) to the motor drive circuit.

- The ASMC-04B Robot Servo motor was used to move exoskeleton arm, which has a voltage $(12 \mathrm{Vdc} \sim$ $24 \mathrm{Vdc})$, and a maximum current of $(3.2 \mathrm{~A})$. This servo motor was converted to DC motor to avoid the limitation in its rotation that found in servo. To be able to control a motor of higher voltage and current than the logical circuit that can handle, a L298N Motor Drive (H-bridge) was used.

- Pulley was connected to the motor shaft supported with a Bowden cable that matches the motor with the actuator, as shown in (Figure 4).

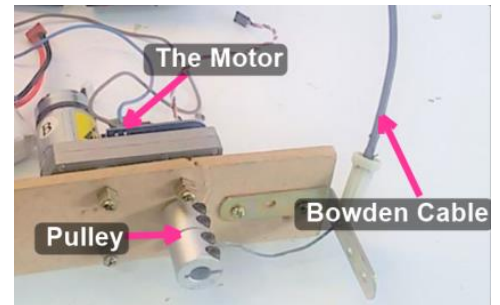

Figure (4): DC motor with pulley and Bowden cable.

- Lithium polymer (LiPo) battery, three cells, $11.1 \mathrm{~V}$, and $2200 \mathrm{~mA}$ was used as a power supply to the exoskeleton device, and it was charged by "B3 pro" three cells charger.

- Gyroscope sensor was connected to the microcontroller and put it in the forearm for measuring the elbow angle as shown in (Figure 5).

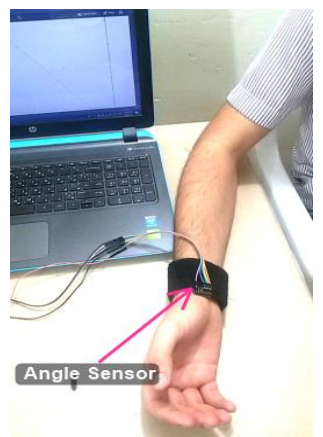

Figure (5): Measuring elbow angle.

- Exoskeleton device is the intrinsic interaction between human and exo-arm. This interaction is twofold. First, cognitive, because the human controls the exoskeleton while it provides feedback to the 
human; second, a biomechanical interaction leading to the application of controlled forces between both of them (the human and the exoskeleton) [17]. So, it is important to take care of the design by selecting a material with appropriate characteristics and designing it professionally to suit this purpose. A metal-arm brace is a product from (I-Ming Sanitary Materials Co. Ltd) was used in this study. It consists from aluminum shafts, stainless steel joint, two bandages, and four straps, (Figure 6).

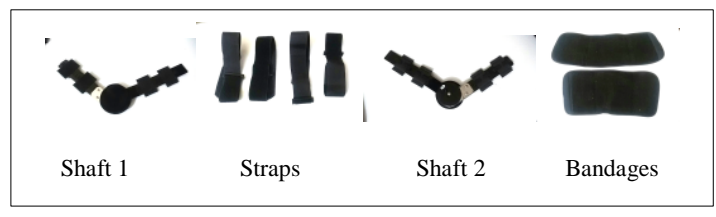

(a)

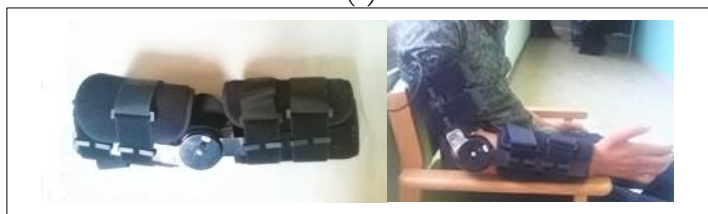

(b)

Figure (6): The present exo-arm design: (a) Component of metal-arm brace, (b) Metal-arm brace wearing.
- This metal arm brace was converted to active orthosis that gives flexion and extension of elbow joint by putting Bowden cable in forearm actuator that, works to flex the exo-forearm toward the exoupper arm like the human flexion muscle of elbow joint, and by putting a resistive spring that works as exo-extension and works as resistant for active-resist mode, see (Figure 7).

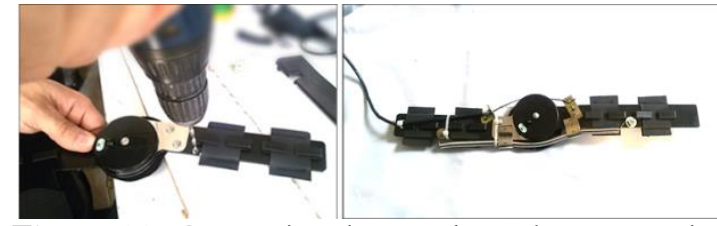

Figure (7): Converting the metal-arm brace to active orthosis.

- For safety:

1. Two limit switches were put to limit the continuous movement of DC motor.

2. The switch was connected to power supply.

3. Switch for running the motor in two directions.

- Microcontroller, motor, motor driver, switches and battery were kept in a wooden box $(17 \mathrm{~cm} * 23.5$ $\mathrm{cm})$. Figure 8 shows the exoskeleton device with its entire components.

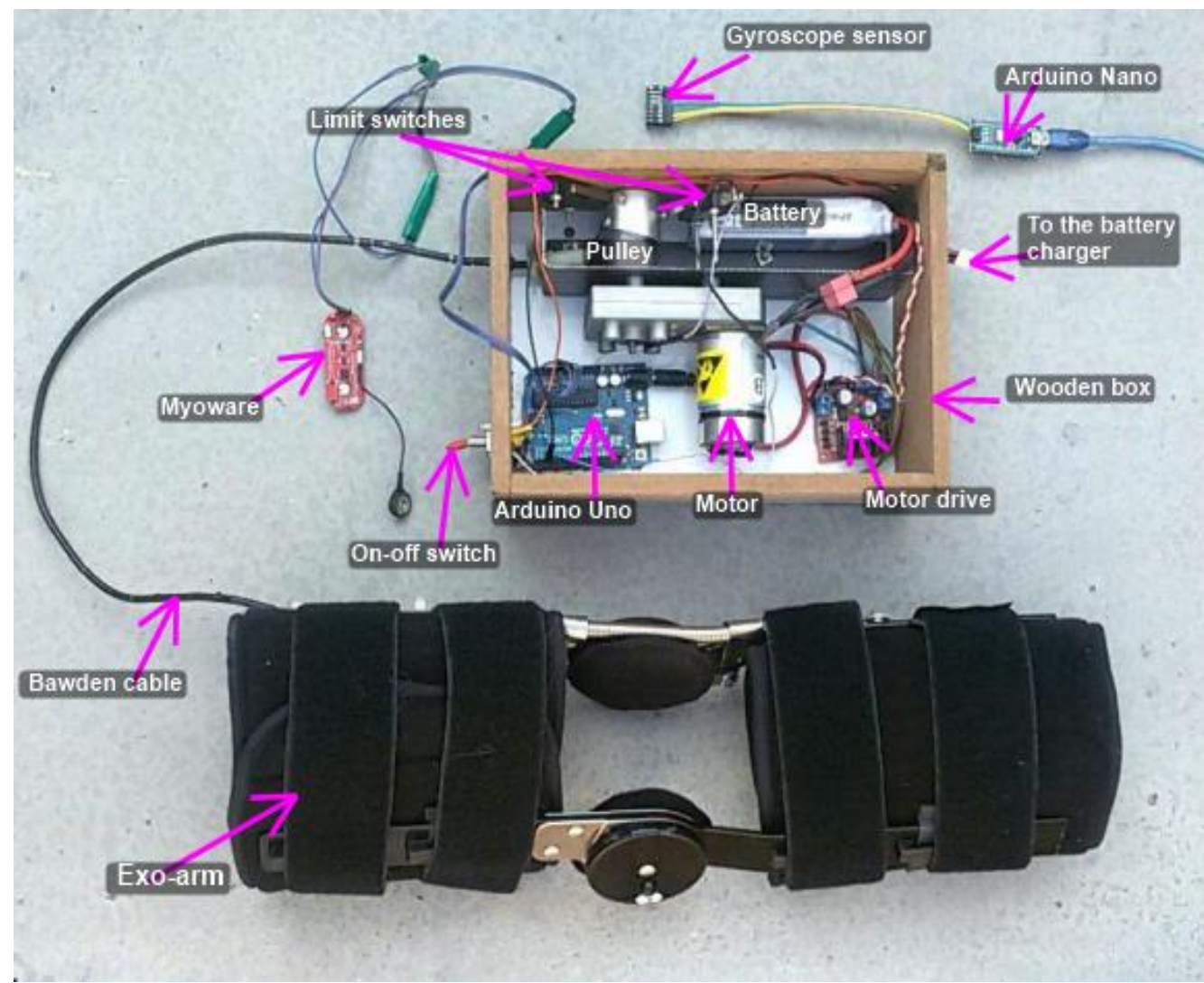

Figure (8): The exoskeleton device with its entire components.

- For software; programming MATLAB and Arduino were used to analyze the EMG signal, control movement of the motor and draw curves of elbow angle.

Testing the robotic exoskeleton, after the exoskeleton-robot device was completely designed, it was tested on 4 healthy subjects according to motor velocity, motor torque, ROM, a lifetime of the battery, and time for charging the battery.

Applying the device to the patients; steps of applying the exoskeleton device on patients have been done in Baghdad teaching hospital, Ghazi al Hariri hospital, medical rehabilitation and arthritis center, and also in patients' homes. 
In this study, the exo-arm was applied to stroke patients to rehabilitate the arm through flexion and extension of the elbow joint (Figure 9). Elbow angle was measured for the healthy arm of the same patient as a reference.

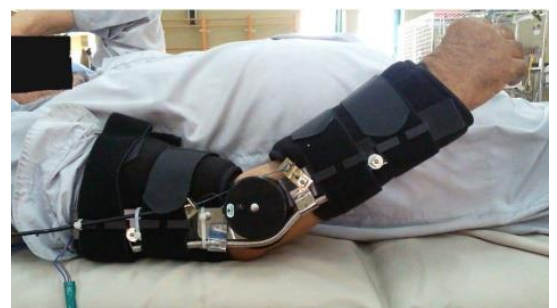

Figure (9): Apply the device to the patient.

Steps of rehabilitation therapy, the first and most important step is that each patient must know the objective and importance of the exoskeleton device. Steps of physiotherapy start with a passive exercise where the patient can't move his arm in the first sessions. Then, passive exercise turns to active exercise when the patient begins to flex his arm weakly.

The EMG signal from biceps brachii muscle was measured at the beginning of each session and according to this signal, the microcontroller was programmed to give the order to the motor driver to start moving. With each session elbow ROM and EMG signal were measured as a biofeedback. Elbow ROM was measured with and without exoskeleton for the affected arm to see how the ROM develops from first to the last session.

This exoskeleton robot was applied to 4 normal volunteers, and 15 stroke patients. Each patient submits to many sessions with an average of two sessions per week. The total number of sessions depends on the severity of the injury or stroke for each patient.

Patient's information was recorded, which includes: (name, gender, age, height, weight, type of disease and medical history). Elbow angle was measured for the normal arm and the affected arm by using goniometer (Figure 10).

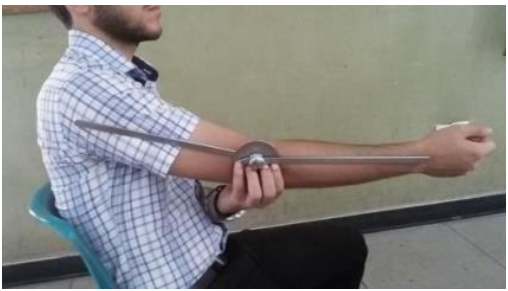

Figure (10): Measuring elbow angle by goniometer.

\section{Results}

Results are for the patient who submits to twenty physiotherapy sessions, two sessions per a week. The first nine sessions are passive exercises and from tenth to twentieth sessions are active exercises. Patient's information present in (Table 1).
Table (1): Patient's Information.

\begin{tabular}{|l|l|}
\hline Name & A.S. \\
\hline Gender & Male \\
\hline Age (year) & 67 \\
\hline Length $(\mathrm{cm})$ & 160 \\
\hline Mass (kg) & 70 \\
\hline Type of disease & Stroke \\
\hline Affected arm & Right \\
\hline $\begin{array}{l}\text { Time between passing the } \\
\text { disease and starting therapeutic } \\
\text { exercises (week) }\end{array}$ & 4 \\
\hline
\end{tabular}

Results include the curves of elbow ROM and the curves of EMG signal.

The first session shown in (Figure 11) indicates to passive exercise, the tenth session shown in (Figure 12) indicates to the first active-assist exercise, the fifteenth session shown in (Figure 13) indicates the first active-resist exercise, and the twentieth session shown in (Figure 14) indicates to the last active-resist exercise.
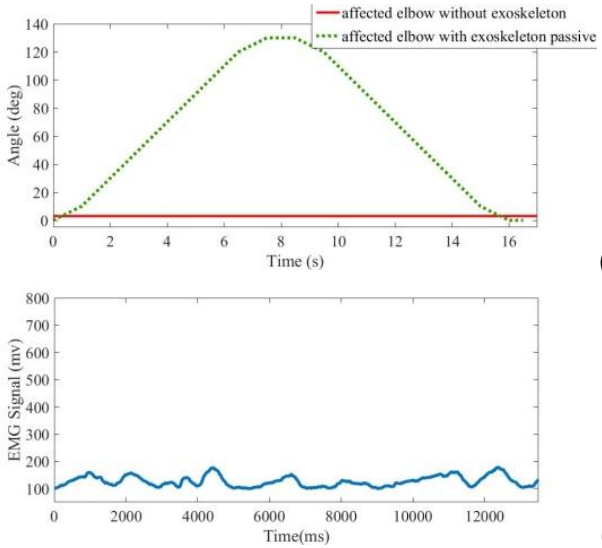

(B)

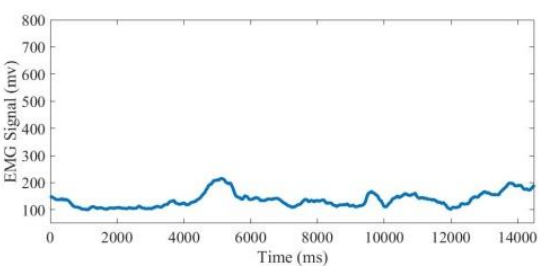

Figure (11): 1st session, passive exercise for: (A) Elbow ROM, (B) Biceps signal without exoskeleton, (C) Biceps signal with exoskeleton.
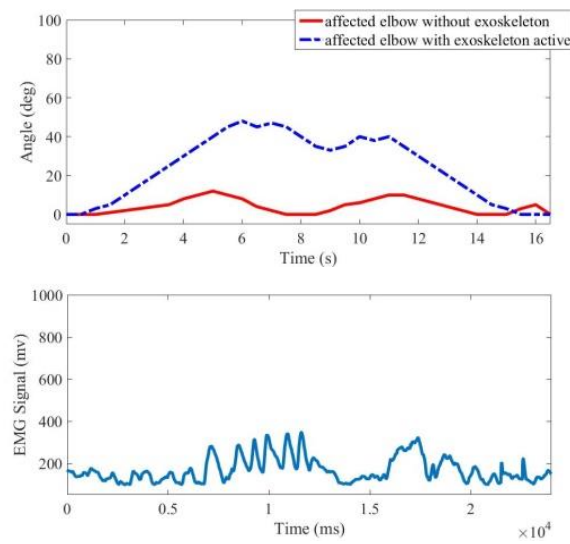

(A) 




Figure (12): 10th session, first active-assist exercise: (A) Elbow ROM, (B) Biceps signal without exoskeleton, (C) Biceps signal with exoskeleton.
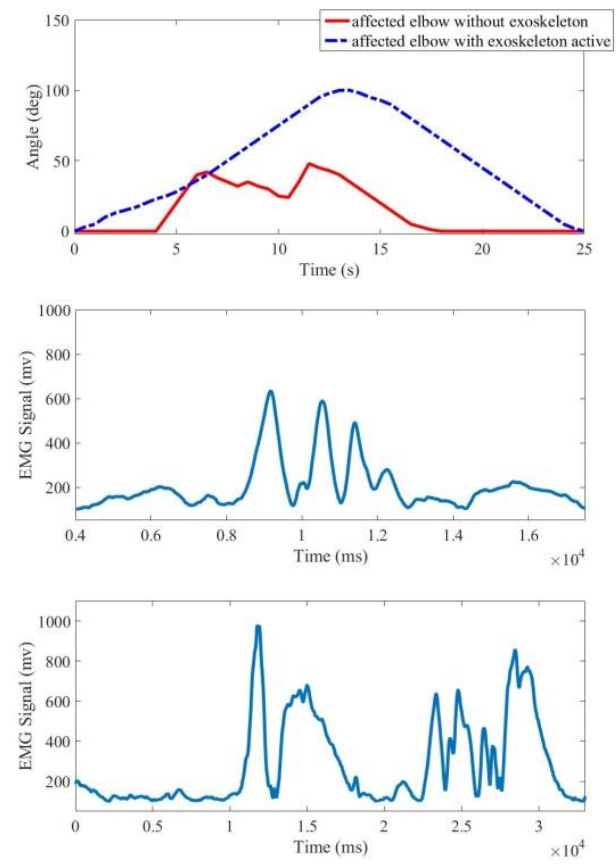

Figure (13): 15th session, first active-resist exercise:

(A) Elbow ROM, (B) Biceps signal without exoskeleton, (C) Biceps signal with exoskeleton.
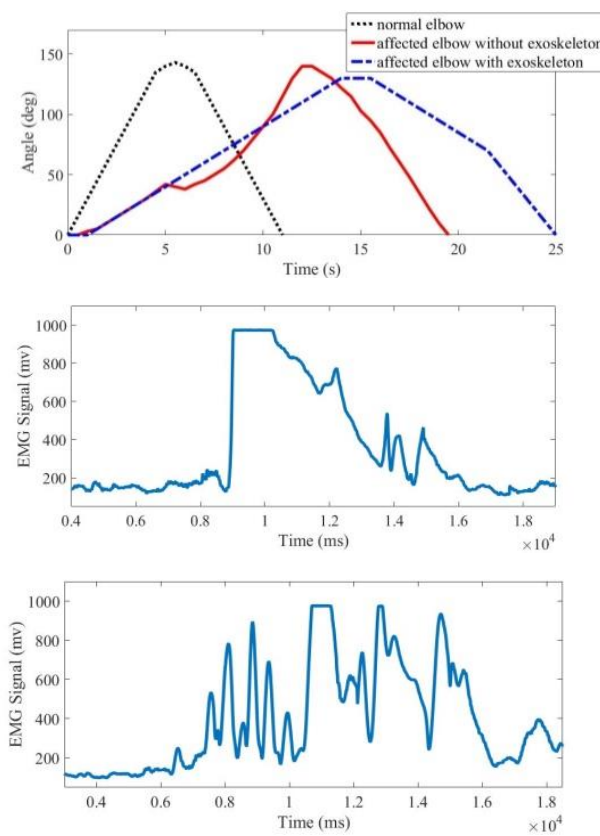

Figure (14): 20th session, last active-resist exercise for (case 2): (A) Elbow ROM, (B) Biceps signal without exoskeleton, (C) Biceps signal with exoskeleton.
Figure 15, shows the development of elbow flexion angle for this patient through first to twentieth sessions when the device is conducting the exercises of physiotherapy.

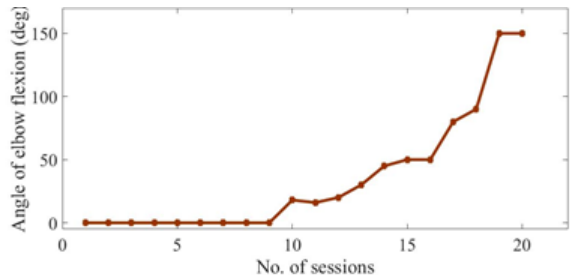

Figure (15): development of elbow angle per sessions.

\section{Discussion}

This study focused to evaluate the influence of a training exercises using exoskeleton for physiotherapy, with a focusing on improvement of the functionality of everyday activities in subjects with lose of movement in the upper limb as a result of stroke.

This exoskeleton device works to perform two kinds of therapeutic exercises: passive exercise and active exercise. It starts with the patient from the first session of physiotherapy, but if the device gives only active exercise, in this case, the therapy will begin from advanced sessions when there is enough muscle force to move weakly. Passive exercise acts as an external force that move the limb, and this movement don't related with muscle force, therefore there is no effect to muscle signal EMG through passive exercise because it is not voluntary movement.

The patients should understand how to regulate and control their muscle activation through the exercise. For that, the use of continuous myoelectric control would have an advantage in exoskeleton-aided stroke physiotherapy as it provides high interaction during the whole movement exercise.

\section{Conclusions}

For this study, the following conclusions can be reached:

- The results showed the importance of using EMG signal as an input control for exoskeleton arm that enhance the outcome of physiotherapy and this occur in EMG biofeedback where the muscle signal show good activity through active exercise, this occur from moving the muscle from its voluntary force.

- The results illustrated a change in the joint angle for the better from first to last session.

\section{Future Work}

The suggestions for future work of this study are listed in the following points:

1. Develop an exoskeleton robot for multi DOF that give rehabilitation for all upper limb joints and muscles.

2. There might be a possibility of using computer assisted system with special software or mobile application that includes interactive process of physiotherapy - exoskeleton procedure. 


\section{References:}

[1] B. Ghannadi, "Model-based Control of Upper Extremity Human-Robot Rehabilitation Systems," Thesis, University of Waterloo, 2017.

[2] C. Fleischer, "Controlling Exoskeletons with EMG Signals and a Biomechanical Body Model," Thesis, University of Berlin, 2007.

[3] K. Jobes, M. Bernier, and S. Dryer, "Arm Mounted Exoskeleton to Mechanically Assist Activities of Daily Living," Program in Electromechanical Engineering, College of Engineering, Wentworth Institute of Technology, Boston, MA, 2016.

[4] C. Mavroidis, "Smart portable rehabilitation devices," J. Neuroeng. Rehabil., vol. 2, no. 1, p. 18, 2005.

[5] M. Cempini, "NEUROExos: A Powered Elbow Orthosis for Post-Stroke Early Neurorehabilitation," Eng. Med. Biol. Soc. (EMBC), 35th Annu. Int. Conf. IEEE, vol. 3-7, no. 13, pp. 342-345, 2013.

[6] R. Cappato de Araújo, D. Rocha, and R. Pitangui, "The Influence of Dynamic Orthosis Training on Upper Extremity Function After Stroke: a Pilot Study," Journal of Healthcare Engineering, vol. 5, no. 1, pp. 55-66, 2014.

[7] C. Bator and R. Svensson, "Exoskeleton Arm: How to Construct a Smart Support Structure for an Arm," Thesis, KTH Royal Institute of Technology, 2016.

[8] S. Ball, "Novel Robotic Mechanisms for Upper-Limb Rehabilitation and Assessment," Thesis, Queen's University Kingston, Ontario, Canada, 2008.

[9] D. Naidu, "Bio-Mechatronic Implementation of a Portable Upper Limb Rehabilitative Exoskeleton," Thesis, University of KwaZulu-Natal, 2011.
[10] E. Artz, "Myoelectric Control of a Robotic Exoskeleton for Rehabilitation," Thesis, Rice University, 2015.

[11] N. Vitiello, "NEUROExos: A Powered Elbow Exoskeleton for Physical Rehabilitation," IEEE Transactions on Robotics, vol. 29, no. 1, pp. 220 235, 2013.

[12] J. Rosen, M. Brand, and M. Fuchs, "A Myosignal-Based Powered Exoskeleton System," IEEE Trans. Syst. Man, Cybern. A Systems and Humans, vol. 31, no. 3, pp. 210-222, 2001.

[13] E. Rocon, J. Belda-Lois, and A. Ruiz, "Design and Validation of a Rehabilitation Robotic Exoskeleton for Tremor Assessment and Suppression," IEEE Transactions on Neural Systems and Rehabilitation Engineering, vol. 15, no. 3, pp. 367-378, 2007.

[14] R. Gopura, D. Bandara, and J. Gunasekara, "Recent Trends in EMG-Based Control Methods for Assistive Robots," chapter twelve of Electrodiagnosis in new frontiers of clinical research, IntechOpen, pp. 237-268, 2013.

[15] R. Chandra, "Development and Control of Upper-Limb Exoskeleton Robots," Thesis, School of Science and Engineering, Saga University, Japan, 2009.

[16] A. Ruiz, A. Forner-Cordero, and E. Rocon, "Exoskeletons for Rehabilitation and Motor Control," in Biomedical Robotics and Biomechatronics, The First IEEE/RAS-EMBS International Conference, pp. 601-606, 2006.

[17] M. Rahman, T. Kittel-Ouimet, and M. Saad, "Development and Control of a Robotic Exoskeleton for Shoulder, Elbow and Forearm Movement Assistance," Applied Bionics Biomechanichs, vol. 9, no. 3, pp. 275-292, 2012. 